\title{
Pesquisa de UX em jogos de Realidade Virtual: desafios e lições aprendidas
}

\author{
Juan Oliveira \\ SIDIA \\ Manaus, Brasil \\ juan.braga@samsung.com
}

\author{
Wilson Prata \\ SIDIA \\ Manaus, Brasil \\ wilson.p@samsung.com
}

\begin{abstract}
RESUMO
O presente trabalho tem como objetivo apresentar problemas e desafios enfrentados ao se avaliar a satisfação de uso de jogos de Realidade Virtual. Utilizamos como objeto a avaliação dos jogos Angest e Dead Body Falls, dois Walking Simulator desenvolvidos pelo Black River Studios. Através da aplicação e adaptação de abordagens qualitativas, conforme descrito, conseguimos tratar dos desafios desse tipo de avaliação. Por fim, listamos os ganhos no nível tático, estratégico e operacional a partir dessa pesquisa e quais as considerações para a área de IHC.
\end{abstract}

Palavras-chave: realidade virtual; avaliação de jogos; walking simulators

\section{DESCRIÇÃO DO PROBLEMA}

Jogos eletrônicos são uma experiência altamente interativa. Ser interativo significa que o sistema de computador deve processar uma série de entradas enviadas pelo jogador (inputs), fornecendo-lhes, como resposta, as saídas que estes jogadores precisam decodificar para avançar no jogo (outputs). Ao dar entrada e decodificar as respostas dadas pelo sistema, o jogador desenvolve sua experiência de jogo. Essa experiência constroe-se a partir da sequência dos eventos que dão significado para todas suas ações dentro do jogo. Caso essa sequência de eventos seja satisfatória, a experiência decorrente pode criar uma profunda imersão do jogador no jogo. Essa imersão é capaz de desencadear fortes emoções sociais. Assim, enquanto o jogador avança no jogo, é possível que os eventos desdobrados produzam raiva, felicidade, tristeza ou mesmo um sentimento de pertencimento [1].

O gênero do jogo é um aspecto fundamental para a imersão

Permission to make digital or hard copies of all or part of this work for personal or classroom use is granted without fee provided that copies are not made or distributed for profit or commercial advantage and that copies bear this notice and the full citation on the first page. Copyrights for components of this work owned by others than the author(s) must be honored. Abstracting with credit is permitted. To copy otherwise, or republish, to post on servers or to redistribute to lists, requires prior specific permission and/or a fee. Copyright 2018 SBC.

IHC 2018, Anais Estendidos do XVII Simpósio Brasileiro sobre Fatores Humanos em Sistemas Computacionais

Outubro 22-26, 2018, Belém, Brasil

IHC na Prática e evolução do jogador dentro da experiência. A depender do tipo de jogo, a ligação emocional entre o jogador e os personagens podem ser mais fortes, favorecendo a imersão. Nesse sentido, os jogos do gênero walking simulator ("simulador de caminhada" em inglês), são um bom exemplo. Os walking simulators, em geral, não apresentam aspectos tradicionais de um jogo (como um objetivo claro, condições de vitória / derrota, rankings, etc). Nesse tipo de jogo, o jogador se depara com uma narrativa de ritmo lento, cobrindo tópicos sobre contemplação e exploração com algum nível de evolução emocional [2] [3].

É ponto pacífico na indústria de tecnologia que um dos pontos fortes da realidade virtual (RV) é sua capacidade de criar experiências a partir da imersão e sensação de presença [4]. No contexto de jogos em RV essas características se intensificam quando se trata de jogos do tipo walking simulator. Evidentemente, walking simulator e jogos em Realidade Virtual têm muitas características comuns e complementares. Algumas dessas semelhanças entre o gênero e a plataforma são: ritmo lento, experiências imersivas e forte vínculo emocional [5]. Estes elementos são decididos pelo Designer do jogo, o profissional responsável por definir todos os elementos centrais da experiência do jogador e quão bem o jogo suporta e fornece o tipo de diversão que os jogadores querem ter [6]. Juntos e bem combinados, walking simulators e RV são recursos fortes para que os Designers de jogos desenvolvam experiências satisfatórias para os jogadores.

Angest e Dead Body Falls são walking simulators em RV desenvolvidos pela Black River Studios, setor de jogos do SIDIA (Samsung Instituto de Desenvolvimento para a Informática na Amazônia)[7][8]. Os jogos foram desenvolvidos para a plataforma Gear VR, dispositivo de realidade virtual da Samsung, pertencente a categoria dos chamados Head Mounted Display (HMD), onde o usuário precisa conectar um smartphone ao aparelho para ter acesso ao conteúdo em RV. 


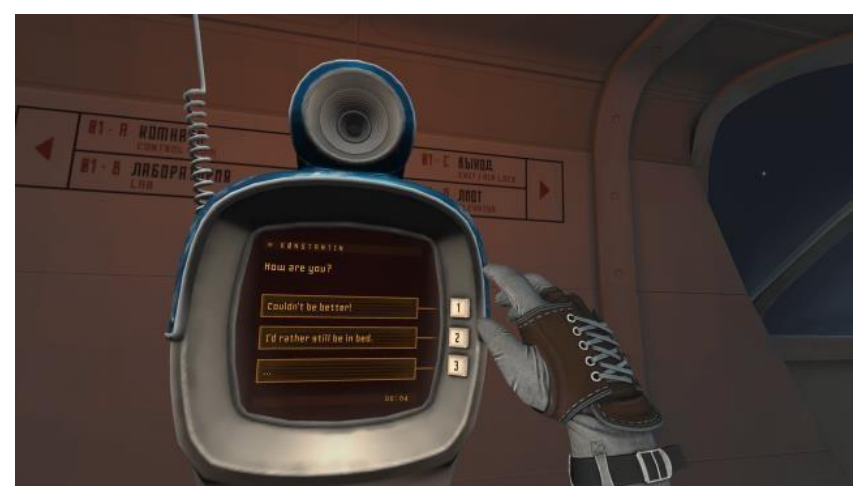

Figura 1 - Captura de tela do jogo Angest

Em ambos os jogos, o jogador é guiado por uma narrativa envolta em mistério, os objetivos não são claros e cabe ao jogador explorar o cenário para interagir com objetos que podem ajudá-lo a entender melhor a história dos jogos, onde a narrativa é usada como um recurso do gameplay. Assim, ambos os jogos carecem, intencionalmente, de muitos elementos que normalmente compõem a experiência do jogador como "diversão em vencer obstáculos", "recompensa intrínseca", "aumento da carga de trabalho" e a premissa de que "humanos precisam ser desafiados"[2].

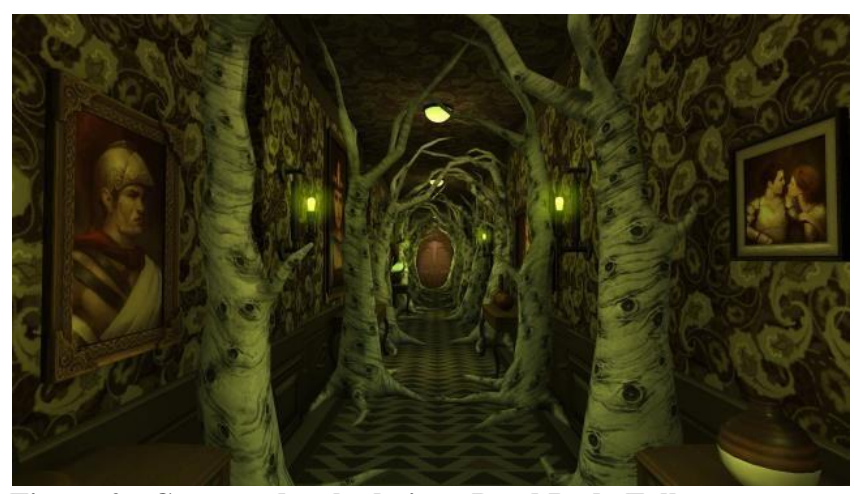

Figura 2 - Captura de tela do jogo Dead Body Falls

Essas particularidades despertam algumas questões interessantes. Já que "escolhas significativas" são um pilar bem conhecido do bom design de jogos [9], uma vez que as escolhas não estão disponíveis para o jogador, as outras decisões no design do jogo compensam essa falta? Se a condição vencedora é um paradigma amplamente usado na indústria do jogo, que tal a experiência do jogador em um jogo que não é possível ganhar ou perder? ... e para a perspectiva de pesquisa, qual é a definição de sucesso em jogos com essa característica?

\section{ABORDAGEM ADOTADA}

Nos dois jogos aqui citados, a maioria das perguntas e dúvidas da pesquisa estavam diretamente relacionadas à satisfação do jogador e à percepção subjetiva da trama e da experiência geral. Dessa forma, optou-se por uma abordagem qualitativa, compartilhada do ferramental metodológico das ciências sociais. Assim, como na maioria das pesquisas de ciências sociais, a metodologia utilizada para avaliar nosso objeto (satisfação do jogador de jogos walking simulator em VR) foi construída com base em questões de pesquisa identificadas junto ao time que desenvolveu o jogo.

Depois que as questões de pesquisa foram identificadas, o próximo desafio foi escolher as técnicas de pesquisa que pudessem lidar com todas essas questões.

A experiência de jogar um jogo de RV mais profundo fornece emoções complexas nos jogadores o que permite que cada um construa sua própria perspectiva dessa experiência. Para selecionar as ferramentas, lembramos que as abordagens subjetivas funcionam melhor para identificar as atitudes e preferências dos jogadores, portanto, era esperado que a entrevista após o teste (debrifing) fosse o principal instrumento para responder às questões de pesquisa. Como não há categorias claras, específicas e dedicadas para avaliar jogos walking simulators, perguntas abertas são mais indicadas do que perguntas fechadas. Dessa forma, a sessão de debriefing foi um ponto chave e o design de perguntas abertas direcionadas para a proposta do jogo funcionou bem com os jogadores. Com isso, o feedback foi natural e rico.

Recrutar participantes foi um aspecto central para o sucesso dos testes, um pequeno questionário foi desenvolvido para garantir que o perfil de participantes corresponde àquele que o jogo almejava alcançar. Além das questões sociodemográficas, a questão principal era sobre o estilo de jogo preferido do participante. Os walking simulators são, em outras palavras, fortes vieses, ou as pessoas odeiam ou as pessoas o amam [3]. Dessa forma, o questionário de recrutamento perguntava os jogos favoritos do potencial participante e se ele /ela já tinha jogado algum jogo do gênero em questão. Só participaram da pesquisa jogadores que apreciavam esse tipo de jogo. Essas perguntas mostraram-se apropriadas para validar o perfil do jogador.

Os testes dos jogos seguiram a estrutura geral da maioria dos testes de validação com o usuário: um moderador e um facilitador deram as boas-vindas aos participantes; eles foram solicitados a assinar um formulário de acordo de confidencialidade; as instruções foram apresentadas para os participantes; eles jogaram o jogo; houve o debriefing e, em seguida, cada participante foi agradecido recompensado pela sua participação.

O planejamento, a execução e o relatório dos resultados levaram quatro semanas ao todo. $\mathrm{O}$ passo a passo metodológico foi: i) compreender o desafio e definir questões de pesquisa; ii) definir o perfil do jogador; iii) selecionar metodologias; iv) definir um cronograma; v) recrutar participantes; vi) configurar a localização, documentação e dispositivos do playtest; vii) rodar o playtest; viii) coletar os dados; ix) analisar os dados; $\mathrm{x}$ ) relatório parcial; xi) relatório final e apresentação de resultados.

Nesse sentido, o uso de um documento de pesquisa para organizar o teste, detalhar sua estrutura e resultados 
mostrou-se uma ferramenta relevante para manter o foco e o contexto sempre claros ao longo das quatro semanas.

\section{RESULTADOS}

Os desafios de testar jogos desenvolvidos para tecnologias emergentes como a Realidade Virtual, são muitos, sendo o primeiro deles, a busca por usuários com alguma experiência de uso e que não se mostrem tão "encantados" com a tecnologia. Para contornar esse problema, costumamos testar nossos jogos em Arcades de Realidade Virtual, já bastante comuns na cidade de São Paulo. Nesses lugares, conseguimos encontrar um público familiarizado com essa tecnologia, que costuma jogar jogos RV com certa regularidade e que já experimentou vários jogos nessa plataforma. Por conta disso, esse público tende a ser bastante crítico quanto a qualidade dos jogos, fornecendo opiniões e sugestões que tem ajudado a equipe de desenvolvimento a melhorar os jogos que são testados.

Um outro desafio diz respeito a usabilidade em jogos, uma vez que existe uma fina linha que separam os problemas de usabilidade e a dificuldade inerente ao desafio que o jogador deve enfrentar para dominar o jogo. De forma geral, nossa experiência tem mostrado que os problemas de usabilidade (como falta de consistência ou uso de modelos mentais que desconsideram a bagagem cultural do jogador) costumam interferir na curva de aprendizado do jogador, tornando a experiência de jogo penosa e, por vezes, desengajadora. Para lidar com questões como essa, o profissional que planeja e executa os testes precisa trabalhar alinhado com o Designer de jogos. Em nossas pesquisas de satisfação de jogos, costumamos envolver esses profissionais em todas as fases da pesquisa, desde a definição das questões de pesquisa e hipóteses, até a condução dos testes. Nossa experiência tem mostrado que essa abordagem tem servido para melhorar o engajamento desse profissional e do time como um todo em torno de questões relacionadas a experiência do jogador.

A configuração de teste para jogos de RV é outro desafio técnico, especialmente quando executado em um dispositivo HMD como o Samsung Gear VR. Dispositivos como esses costumam superaquecer, algo que pode acabar perturbando a experiência de jogo e o teste. Nos testes realizados este problema é evitado graças a qualidade do software. Todos os jogos passam pelo setor de QA (quality assurance) para garantir que o dispositivo não vá travar ou desligar por conta de um problema de performance.

O Samsung Gear VR permite compartilhar a tela do jogador com uma smartTV durante a sessão do jogo. Contuto, após um teste piloto, percebeu-se que esse recurso apresentava uma configuração difícil e demorada, além de impactar o desempenho do hardware e da bateria do celular. Assim, optou-se por abandonar essa possibilidade para reduzir o atrito na experiência.

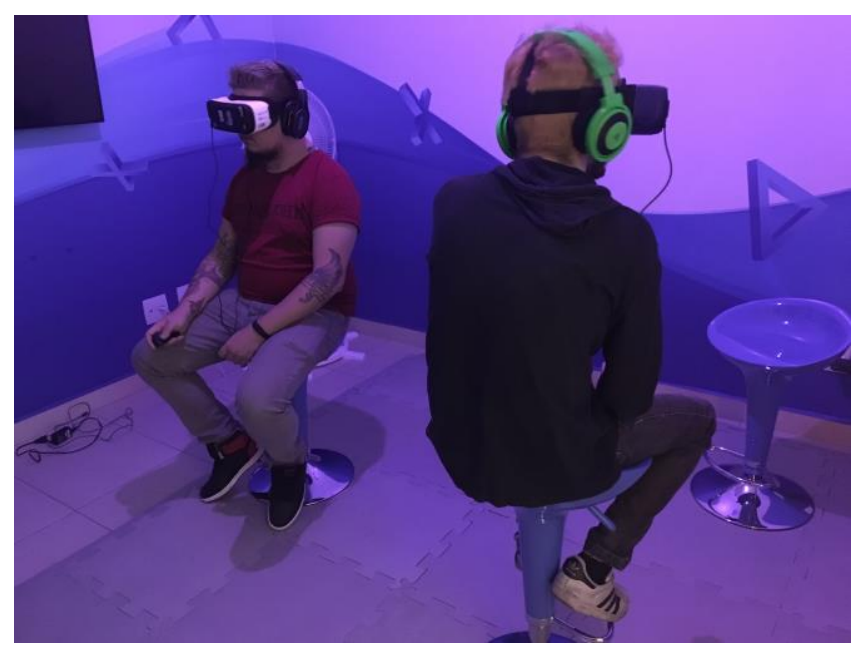

Figura 3 - Sala de playtest de jogo para a plataforma Samsung Gear VR

Em geral, o feedback da equipe de desenvolvimento do jogo sobre os testes tem sido positivo. Na avaliação do jogo Angest, por exemplo, muitas das suposições e hipóteses levantadas puderam ser confirmadas e o relatório foi usado para justificar algumas decisões de mercado e promoção. À primeira vista, desenvolver um walking simulator para RV, tinha se mostrado uma escolha de enorme risco uma vez que se trata de um tipo de jogo de nicho. Todavia a avaliação subjetiva dos jogadores confirmou que essa escolha poderia se tornar uma boa decisão estratégica. Os comentários sobre a loja Oculus e outras revistas especializadas [10] foram muito semelhantes e aos coletados durante as avaliações com os jogadores, o que acabou reforçando os resultados e a metodologia da pesquisa. A pesquisa também ajudou a definir a expectativa de sucesso do jogo para a alta gerência da empresa.

\section{CONCLUSÃO}

Avaliar a experiência do jogador em jogos de RV do tipo walking simulator trata-se de um tipo de pesquisa que exige planejamento, organização e principalmente alinhamento entre o time de desenvolvimento e a equipe de pesquisa. É extremamente necessário que a equipe de pesquisa compreenda a proposta do jogo, a mecânica e as intenções de seus criadores (Designer de jogos). A realidade virtual não é tão familiar quanto muitas outras plataformas, como desktop e dispositivos móveis, mas está crescendo rapidamente em muitas áreas diferentes, não apenas em jogos. Assim, é importante que os jogos e as pesquisas sobre UX estejam preparados para lidar com as especificidades dessa tecnologia, de modo que esperamos que esse trabalho ajude à novos pesquisadores e interessados em RV a estarem preparadas para esse desafio.

Nossa percepção é que, quando o objeto de pesquisa é um novo desafio em um dado campo, o primeiro procedimento é retornar à base do campo, rever seus fundamentos e verificar quão relevantes eles permanecem. Em nossas pesquisas costumamos selecionar técnicas de Experiência 
do Usuário (UX) e da área de Design de jogos. Há um ganho duplo com essa estratégia, i) primeiro, embora a base de um campo às vezes não seja suficiente para esgotar todas as dúvidas em uma pesquisa, elas são abertas e genéricas o suficiente para permitir que a pesquisa comece a abordar o problema; ii) segundo, é possível esclarecer o quanto o objeto da pesquisa ainda pertence àquele dado campo de conhecimento. Caso essa relação entre conhecimento do campo e pertencimento do objeto ao campo seja muito fraca, torna-se evidente a necessidade de buscar novas abordagens e métodos de outros campos do conhecimento.

O corpus teórico da Interação Humano-Computador ainda se mostra como o ponto de partida que oferece o ferramental teórico para os pesquisadores de tecnologias emergentes. Contudo, seu arcabouço não é suficiente para resolver todos os desafios que têm surgido. Dessa forma, espera-se que em um futuro próximo, a avaliação de jogos de RV tenha seus próprios métodos e aparatos. Até lá, para ajudar nessa construção, faz-se necessária a aplicação e depuração dos métodos já legitimados em campos correlatos.

\section{REFERENCIAS}

1. Prata W., Oliveira J., Melo P. (2018) Walking with Angest: Subjective Measures for Subjective Evaluation in a Walking Simulator Virtual Reality Game. In: Chen J., Fragomeni G. (eds) Virtual, Augmented and Mixed Reality: Applications in Health, Cultural Heritage, and Industry. VAMR 2018. Lecture Notes in Computer Science, vol 10910. Springer, Cham

2. Moyama, et al.: Games user research (GUR): our experience with and evolution of four methods. In: Isbister, K., Schaffer, N. (eds.) Game Usability: Advice from the Experts for Advancing the Player Experience. Elsevier Inc. (2008)

3. PC Games: Talking 'walking sims': The Chinese Room's Dan Pinchbeck on the pointlessness of the debate. https://www.pcgamesn.com/dear-esther/danpinchbeck-interview-are-walking-sims-games. Acessado em 18 Fev 2018

4. M. Abrash, What VR Could, Should, and Most Certainly Will Be Within Two Years. Presentation Notes, 2014. https://www.youtube.com/watch?v=G2dQoeqVVo. Acessado em 18 Fev 2018

5. Lazzaro, N.: The four fun keys. In: Isbister, K., Schaffer, N. (eds.) Game Usability: Advice from the Experts for Advancing the Player Experience. Elsevier Inc. (2008)
6. Rolling Stones: Why VR Is the Next Step for Walking Simulator

Games. https://www.rollingstone.com/culture/news/vr-is-thenext-step-for-walking-simulator-games-w445685. Acessado em 19 Fev 2018

7. Black River Studio, http://blackriverstudios.net/angest/, Acessado em 19 Fev 2018

8. Black River Studio, http://blackriverstudios.net/deadbodyfalls/, Acessado em 30 Jul 2018

9. Isbister, K. How Games Move Us. iBooks (2017)

10. Mapinguari Nerd: Review: Angest - uma breve análise de tempo | memórias de um old gamer. http://mapinguanerd.com.br/angest-uma-breve-analisede-tempo-memorias-de-um-old-gamer/. Accessado em: 12 Jan 2018 